Conclusion: Changes in endurance shuttle walking performance, especially improvements, are well perceived by patients with COPD. From this set of data, it should be possible to identify the smallest change in walking performance with a high likelihood of being perceived by the patients (MCID).

\section{Aerobic Training with Hyperoxia in Patients with Mild/Severe Brain Injury}

\section{Busilacchi Élise, Geneviève Cadoret, Alain-S Comtois, Département de kinanthropologie, UQAM, Montréal, Qc}

Background: Previous studies have shown that aerobic training can induce physiological benefits for brain injured patients. However these benefits seem to be limited. Compared with untrained subjects who follow the same training program, patients with brain injury have a reduced exercise capacity and have difficulty in reaching a high intensity exercise level. A possible alternative to help these patients is to conduct the training in hyperoxia $\left(\mathrm{O}_{2}\right.$ supply). Studies with untrained subjects have shown that training in hyperoxia enhances the fitness performance. Thus, the purpose of this study was to measure the effects of an aerobic training in hyperoxia with brain-injured patients.

Methods: Twelve patients were randomly assigned to either one of two aerobic training protocols: A) 100\% $\mathrm{O}_{2}$; B) $20.9 \% \mathrm{O}_{2}$ (room air). In both protocols training was on stationary bicycle, 2 sessions per week over 10 weeks. Each session lasted $25 \mathrm{~min}$ and was composed of 6 intervals with intensity ranging from 60 to $85 \%$ of the max HR. The first interesting result is that all patients were able to reach and sustain the $80-85 \%$ level of exercise. To measure the working capacity during the training, we recorded the workload (watts) reached at the third interval (80-85\% of the HRmax) of each session.

Results: A difference $(P<0.05)$ in the power output on the ergocycle was observed between the pre test (measured on the first week) and the post test (measured on the 6th week) for subjects trained in hyperoxia but no difference was observed for subjects trained in normoxia. However, no difference was ob- served in the 6 min walk test for all subjects before and after the aerobic training. There was no difference in oxygen consumption (submaximal test $85 \%$ HRmax) between the pre and post tests in both groups of subjects $(P=0.18)$. Nonetheless, subjects trained in hyperoxia showed a $16 \%$ average increase in their $\mathrm{VO}_{2}(85 \%$ HRmax) compared with subjects in normoxia who had a $4 \%$ average decrease.

Conclusion: Our results show that oxygen supply seems to accelerate the benefits of the aerobic training with brain injured patients. We believe that it is the combination of the training protocol (i.e. intervals) and the oxygen addition that induced the early benefits observed.

\section{Chronic Obstructive Pulmonary Disease (COPD) as a Risk Factor for Glucose Metabolism Perturba- tion and Insulin Resistance.}

\section{Doucet M, Laviolette L, Gagné-Belley D, Maltais F, Centre de Recherche, Hôpital Laval, Institut uni- versitaire de cardiologie et de pneumologie de l'U- niversité Laval, Québec, Canada.}

Background: High body mass index (BMI) is associated with better survival in COPD. However, increased BMI and especially waist circumference is associated with elevated pro-inflammatory systemic markers that might contribute to glucose intolerance. On the other hand, COPD is a chronic inflammatory disease that could be a risk factor for impaired glucose metabolism. The objective of this study was to compare the prevalence of glucose intolerance in COPD patients and control subjects with high waist circumference.

Methods: Eleven patients with COPD (age:68 $\pm 8 \mathrm{yr}$ mean $\pm \mathrm{SD} ; \mathrm{FEV}_{1}: 49 \pm 17 \%$ pred) and 10 control subjects (C) (age:63 $\pm 6 \mathrm{yr}$ ) underwent a $75 \mathrm{~g}$ oral glucose tolerance test (OGTT). All subjects had a waist circumference $>102 \mathrm{~cm}$ and no previous history of diabetes. Height and weight were measured and each subject underwent dual-energy X-ray absorptiometry (DEXA) to evaluate fat-free mass (FFM) and fat mass (FM) and abdominal tomography to evaluate visceral fat (VF). Blood samples were taken to measure 